\title{
The effects of dietary Microcystis aeruginosa and microcystin on the copepods of the upper San Francisco Estuary
}

\author{
KEMAL A. GER*, SWEE J. TEH ${ }^{\dagger}$, DOLORES V. BAXA ${ }^{\dagger}$, SARAH LESMEISTER $^{\dagger}$ AND CHARLES \\ R. GOLDMAN* \\ *Department of Environmental Science and Policy, University of California, Davis, CA, U.S.A. \\ ${ }^{\dagger}$ Department of Anatomy, Physiology and Cell Biology, School of Veterinary Medicine, University of California, Davis, CA, \\ U.S.A.
}

\section{SUMMARY}

1. Increasing blooms of Microcystis aeruginosa have unknown impacts on the copepods Eurytemora affinis and Pseudodiaptomus forbesi, which are the dominant zooplankters and key prey species for endangered larval fish in the upper San Francisco Estuary.

2. Laboratory feeding experiments were designed to measure the effect of Microcystis on copepod survival and to distinguish the effects of toxicity and nutrition. In a series of survival tests, copepods were fed a mixed diet of algae plus one of two strains of Microcystis, either producing (MC+) or lacking microcystin (MC-).

3. Microcystis significantly reduced survival even when it was a small proportion of the diet, indicating that toxicity was the major cause of mortality. Contrary to expectation, however, the $\mathrm{MC}+$ strain did not result in higher mortality, suggesting that non-MC metabolites of Microcystis can be toxic to copepods.

4. Across treatments, survival of $P$. forbesi was greater than that of E. affinis, although the two copepods responded differently to both the ratio and the strain of Microcystis in their food. Survival of $P$. forbesi was greater on the $\mathrm{MC}+$ strain and was inversely proportional to the ratio of dietary Microcystis (MC+ or MC-). In contrast, survival of E. affinis declined similarly across treatments and was not related to the proportion or strain of dietary Microcystis. Results indicate that the copepod P. forbesi can coexist with Microcystis while the other copepod E. affinis cannot.

5. Regardless of species, dietary Microcystis caused significant mortality to copepods, and it may cause adverse impacts to the potentially food-limited zooplankton community of the San Francisco Estuary. These impacts may not be related to the cellular MC concentration because Microcystis contains other metabolites that negatively affect copepods.

Keywords: copepod, harmful algal bloom, microcystin, Microcystis, San Francisco Estuary

Correspondence: Swee J. Teh, Department of Anatomy, Physiology, and Cell Biology, Aquatic Toxicology Program, School of Veterinary Medicine, 1321 Haring Hall, One Shields Avenue, University of California, Davis, CA 95616, U.S.A.

E-mail: sjteh@ucdavis.edu

\section{Introduction}

Global increases in the extent of bloom-forming cyanobacteria have attracted growing attention from both management and scientific interests because of potential negative impacts on water quality and food web structure (Kirk \& Gilbert, 1992; Chorus \&

(C) 2010 Blackwell Publishing Ltd 
Bartram, 1999). Microcystis is a common bloom-forming cyanobacterium that can dominate nutrientenriched freshwaters including upper reaches of estuaries, such as the San Francisco Estuary (Paerl, 1988; Lehman et al., 2008).

In blooms, Microcystis can change the food web because it is a poor quality food for zooplankton, reducing their fitness and restricting the trophic transfer of carbon (Paerl, 1988; Christoffersen, 1996; Chorus \& Bartram, 1999). Negative impacts on zooplankton occur in response to a combination of factors including: (i) secondary metabolites with toxic effects, such as microcystins (MCs), (ii) nutritional inadequacy and (iii) poor digestibility or feeding inhibition (Fulton \& Paerl, 1987; DeMott \& Moxter, 1991; Lurling, 2003). However, not all zooplankton are affected equally (Christoffersen, 1996; Wilson, Sarnelle \& Tillmanns, 2006). Species-specific adaptations in zooplankton lead to variation in the observed responses to Microcystis blooms (Kirk \& Gilbert, 1992; Rohrlack et al., 1999; Gustafsson \& Hansson, 2004). Although many studies have evaluated the interaction between zooplankton and Microcystis, most focus on cladocerans while impacts to copepods remain poorly understood (reviewed in Wilson et al., 2006).

Controlled manipulations of diets in the laboratory can provide valuable evidence of the mechanisms involved. A major problem in predicting the impacts of Microcystis, however, has been because of the use of oversimplified experimental conditions, such as pure or single strain diets (presence/absence experiments), a disproportionate focus on daphniids as the test organism, and species/strain-specific responses, preventing the interpretation of impacts on other zooplankton or under field conditions (reviewed in Wilson et al., 2006). As a result, the effect of Microcystis blooms on copepods and the dynamics of food webs in estuaries dominated by copepods are poorly understood.

Copepods are an important link between primary production and fish, and commonly dominate the mesozooplankton of estuaries (Turriff, Runge \& Cembella, 1995; Sommer et al., 2007). Calanoid copepods, particularly Eurytemora affinis (Poppe, 1880) and Pseudodiaptomus forbesi (Poppe \& Richard, 1890), are the principal food source of the endangered larval and pelagic fish in the San Francisco Estuary, where the abundance of pelagic organisms, including copepods and nutritious phytoplankton, has declined to values not seen before (Sommer et al., 2007). Increasing blooms of Microcystis in the freshwater and low salinity zone of the estuary raise concerns about the further depletion of high-quality fish food (MullerSolger, Jassby \& Muller-Navarra, 2002; Lehman et al., 2005, 2008). Understanding the impacts of Microcystis on the dominant copepods of the San Francisco Estuary has become highly significant in the management of its declining fish populations (Sommer et al., 2007).

While a pure diet of Microcystis reduces fitness and survival in zooplankton, it is not likely to occur in nature (DeMott, Zhang \& Carmichael, 1991; Kumar, 2003; Wilson et al., 2006). Unlike daphniids, copepods can ingest nutritious particles selectively and avoid those that are unwanted or toxic (DeMott et al., 1991; Kleppel, 1993; Burns \& Hegarty, 1994; Engstrom et al., 2000; Panosso et al., 2003). Even daphniids show different responses to Microcystis when offered in mixed versus pure diets (Rohrlack et al., 1999; Lurling, 2003). Therefore, in studying interactions between zooplankton and Microcystis, we need to experiment with diets consisting of mixtures of alternative, more palatable, food (Wilson et al., 2006).

Microcystis toxicity comes from well-known toxins, such as MC, but also from other, poorly understood and often unidentified, secondary metabolites (Lurling, 2003; Rohrlack et al., 2004; Wiegand \& Pflugmacher, 2005; Wilson et al., 2006). Though the significance of metabolites other than MC is becoming clear, the relative toxicity to zooplankton of MC versus non-MC metabolites remains unresolved (DeMott et al., 1991; Kurmayer \& Juttner, 1999; Lurling, 2003; Rohrlack et al., 2004). It is possible to quantify the effect of MC in the zooplankton diet using Microcystis strains of identical morphology (assuming similar nutritional profile) but different MC cellular content. This approach has been used successfully to distinguish the toxicity of MC versus non-MC metabolites in daphniids (Rohrlack et al., 1999; Lurling, 2003). When offered in a mixed diet, strains of Microcystis containing (MC+) and lacking (MC-) both caused mortality, showing the toxic effects of non-MC metabolites on daphniids (Lurling, 2003). The current study was designed to compare the effects of Microcystis and its toxic metabolites on the dominant copepods of the San Francisco Estuary.

Our objective was to evaluate the impact of Microcystis in the diet on the survival of the dominant 
copepods. To distinguish between the effects of toxic metabolites and nutritional deficiency of Microcystis on copepods, mixed diets containing nutritious algae and Microcystis (MC+ or MC-) were offered to copepods in laboratory survival tests. Experiments were designed to compare the survival of E. affinis and $P$. forbesi as a function of dietary Microcystis (MC+ versus MC-) and food quantity. We hypothesised that (i) both strains of Microcystis would negatively affect survival, though the response might be species specific, (ii) survival with the MC+ strain in the diet would be lowest and (iii) survival would be inversely proportional to the proportion of Microcystis in the diet.

\section{Methods}

\section{Microcystis aeruginosa strains}

Cultures of two different strains of M. aeruginosa (Kutzing, 1846) were maintained for the feeding experiments in a modified ASM-1 medium (Reynolds \& Jaworski, 1978). MC producing (MC+, UTEX 2385) and MC absent (MC-, UTEX 2386) strains of Microcystis were obtained from the University of Texas Culture Collection (Austin, TX, U.S.A.). Exponentially growing axenic batch cultures were maintained by dilution with freshly prepared cultured medium to keep cell density between $1 \times 10^{6}$ and $3 \times 10^{6}$ cells $\mathrm{mL}^{-1}$, in gently bubbled 500-mL glass flasks, under a $16: 8 \mathrm{~L}: \mathrm{D}$ cycle under $30 \mu \mathrm{mol}$ quanta $\mathrm{m}^{-2} \mathrm{~s}^{-1}$, in a temperaturecontrolled room $\left(22 \pm 1{ }^{\circ} \mathrm{C}\right)$. Under these conditions, both strains were uni- and bi-cellular, the average growth rate of Microcystis was $0.32( \pm 0.04)$ day $^{-1}$ and cell diameter was approximately $4 \mu \mathrm{m}( \pm 0.7)$ for both strains. We assumed that the only difference between the two strains used was MC content and that each strain had a comparable nutritional profile and digestibility.

\section{Instant algae (IA)}

Commercially available and highly nutritious pure phytoplankton food composed of Nannochloropsis and Pavlova, rich in fatty acids, were kept fresh at $4{ }^{\circ} \mathrm{C}$ (Reed Mariculture, Campbell, CA, U.S.A.). Because IA is in a fresh but non-living state, cells have a constant density. Cell diameter was approximately $2 \mu \mathrm{m}$ for Nannochloropsis and $4 \mu \mathrm{m}$ for Pavlova.

\section{Copepod cultures}

Copepods were collected from Rio Vista and Suisun Marsh in the San Francisco Estuary using a 174- $\mu \mathrm{m}$ zooplankton tow net. Pseudodiaptomus forbesi was collected in June 2007 and E. affinis in April 2007 (respective periods of dominance), and returned to the laboratory within $2 \mathrm{~h}$ of collection. Gravid females were selected and rinsed three times with salinityadjusted culture medium before transferring them to the culture. We used reconstituted water (modified by $\mathrm{NaCl}$ to increase salinity) as the culture medium (Horning \& Weber, 1985). Cultures were grown in aerated 4-L beakers placed in an environmental water bath, at $24 \pm 1{ }^{\circ} \mathrm{C}$ for $P$. forbesi and $20 \pm 1{ }^{\circ} \mathrm{C}$ for E. affinis. Water quality in beakers, including dissolved oxygen $\left(>8 \mathrm{mg} \mathrm{L}^{-1}\right)$, $\mathrm{pH}(8.2 \pm 0.1)$, water hardness $\left(100 \mathrm{mg} \mathrm{L}^{-1}\right)$, salinity (5.0 ppt) and ammonia $\left(<1 \mu \mathrm{g} \mathrm{L}{ }^{-1}\right)$, was monitored weekly (Hach, Loveland, CO, U.S.A.). For optimal culture and population growth under laboratory conditions, a previously determined amount of the IA mixture (an equal biovolume of Nannochloropsis and Pavlova) was given

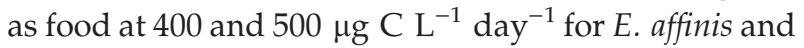
$P$. forbesi, respectively. Approximately, $60 \%$ of the total culture medium was replaced weekly with aerated and temperature-adjusted medium. Copepod culture density was monitored weekly, maintained between 50 and 100 adults $\mathrm{L}^{-1}$, and diluted as necessary to ensure exponential population growth and minimise overcrowding. The systems were maintained under a natural photoperiod $(16 \mathrm{~L}: 8 \mathrm{D})$ and covered with a semitransparent black tarpaulin. The brood cultures were acclimated to these conditions for a minimum of 4 months before the feeding experiments.

\section{Cell size, density and algal biomass determination}

Algal biomass concentration ( $\mu \mathrm{g} \mathrm{C} \mathrm{mL}^{-1}$ ) for the IA mixture and Microcystis was determined by a calibration curve of total carbon and biovolume. A serial dilution of algal biovolumes were filtered on pre-combusted 25-mm GF/C (Whatman, Kent, U.K.), desiccated at $50{ }^{\circ} \mathrm{C}$, and stored at $-80^{\circ} \mathrm{C}$ until the total carbon was measured by a gas chromatogram (UC Davis Stable Isotope Facility, U.S.A.). Microcystis cell size and density was determined using an inverted compound epifluorescent microscope (2007 Zeiss Axio Observer A1, Thornwood, NY, U.S.A), counted in a Haemocytometer 
(Hausler Scientific, Horsham, PA, U.S.A.) and calibrated against light absorbance at $800 \mathrm{~nm}$.

\section{Survival test}

We used a series of survival tests in which dietary treatments included Microcystis while controls did not. The treatment diets consisted of a Microcystis - IA mixture, with the proportion of Microcystis at 0, 10, 25, 50 or $100 \%$ of total food (by carbon), using either the $\mathrm{MC}+$ or MC- strain of Microcystis, plus IA, to a total food concentration of 400 (E. affinis) and 500 (P. forbesi) $\mu \mathrm{g} \mathrm{C} \mathrm{L}{ }^{-1}$ day $^{-1}$. The control diets were comprised of IA only at the same amount as in the associated treatment of IA and Microcystis mixture, which corresponded to $100,90,75,50$ and $0 \%$ (starvation) of the total food. This design allowed the distinction of impacts because of food quantity (total nutrition available) versus quality (MC and non-MC toxicity) by comparing survival over a range of a given diets with and without Microcystis. This design also enabled the assessment of the impact of alternative food sources when Microcystis was present in food.

Experiments took place in triplicates over 11 days in 1-L glass beakers, starting with 10 copepods per beaker and maintained under identical conditions described for the brood cultures. Animals were obtained by growing a new cohort of adults from nauplii in the brood cultures. Experimental copepods were then collected from the pool of $>400$ copepods in the new cohort by gently filtering in a Petri dish for each set of experiments. To minimise the chance of age-related mortality and stress over the 11 days, only active $C_{5}$ copepodites and young adults were randomly selected. Survival and activity were monitored daily using a light table and a dissecting microscope when necessary. Copepods were considered dead only if they showed no sign of movement after disturbance under a dissecting microscope; dead copepods were removed as soon as recorded.

\section{$M C$ verification of strains and treatments}

Microcystis cultures were sampled for MC analysis during the experiment, to ensure the $\mathrm{MC}+$ and $\mathrm{MC}-$ strains were uncontaminated and to measure $\mathrm{MC}$ concentration of the $\mathrm{MC}+$ strain culture. In addition, the MC+ experimental beakers were sub-sampled at day 7 of the survival test for total and dissolved MC
Dietary effects of Microcystis on copepods

1551

analysis to ensure that mortality was not because of any dissolved MCs. A commercially available MC specific competitive ELISA assay with a detection range of $0.16-2.5 \mu \mathrm{g} \mathrm{L}^{-1} \mathrm{MC}-\mathrm{LR}$ was used for MC analysis (Envirologix, Portland, ME, U.S.A.). Microcystis cultures were sampled and kept frozen until analysis, upon which they were thawed to room temperature, sonicated, diluted and measured for MC concentration using a microplate reader at $450 \mathrm{~nm}$. Dissolved MC in treatments was measured 7 days during the experiment, from whole water samples by separating algal cells on a GF/F glass fibre filter (Whatman Corp) and comparing MC concentration in the filtrate with an unfiltered sample. Additionally, a standard polymerase chain reaction (PCR) using published primers designed for the conserved Microcystis-specific $16 \mathrm{~S}$ ribosomal DNA (rDNA) region and amplification of the MIC and MC synthetase genes $m c y \mathrm{~B}$ and $m c y \mathrm{D}$ was used to verify the identity of the two strains of Microcystis. Ability to produce MC is based on the presence or absence of the synthetase genes (Ouellette \& Wilhelm, 2003). Conventional PCR was conducted to verify the presence of Microcystis and the $\mathrm{MC}$ synthetase genes $m c y \mathrm{~B}$ and $\mathrm{D}$ using specific PCR primer sets targeting $16 \mathrm{~S}$ rDNA and conditions described in Ouellette \& Wilhelm (2003). The Microcystis strains were processed for genomic DNA extraction using a modified phenol-chloroform procedure (Sambrook \& Russell, 2001).

\section{Qualitative confirmation of Microcystis ingestion}

To verify ingestion, the presence of Microcystis cells in copepod guts was determined in a separate feeding experiment. Copepods were starved for $5 \mathrm{~h}$ to evacuate gut contents before the experiment, which took place under the same conditions as the survival tests. Copepod density was kept at 10 adults in a 1-L beaker with clean media, and then fed the same treatment diets (MC+ and $\mathrm{MC}-$ ) described earlier. Following a 30-min feeding period, animals were gently filtered, rinsed three times to remove any attached Microcystis cells and narcotised immediately with carbonated water. For each treatment diet, 10 copepods were dissected to expose the gut under a dissecting microscope. Guts were observed for phycocyanin fluorescence (a pigment specific to cyanobacteria), as a qualitative indication of Microcystis cells in copepod guts, using an epifluorescent microscope. 


\section{Statistical analysis}

The effect of diet type on the survival of each copepod species was evaluated by a parametric survival analysis which modelled survival with a Weibull distribution curve (JMP version 7.0; SAS, Cary, NC, U.S.A). Individual probability of mortality or survival (expected proportion of the population dead or surviving) for each treatment and species was calculated by a test of deviance based on the mean number of surviving copepods on the last day of the experiment. Then, the effect of diet type (e.g. Microcystis versus no Microcystis) and copepod species on survival was calculated to compare differences in the effects of the treatments and controls. Finally, the Microcystis only treatments were further broken down to strain (MC+ or MC-) and ratio of dietary Microcystis (\% Microcystis) to analyse differences in the effect of these parameters on copepod survival. Time to $50 \%$ mortality was analysed by linear regression of individual replicate survival responses and calculating when $50 \%$ mortality occurred on average per treatment. All differences noted are significant at $P=0.05$ unless otherwise mentioned.

\section{Results}

\section{Survival test}

Both dietary composition and quantity had very pronounced effects on copepod survival. The presence and proportion of Microcystis drastically reduced survival in all treatments for either copepod species, regardless of Microcystis strain and MC content (Fig. 1). In contrast, survival with the control diets was significantly higher than corresponding Microcystis treatments (Fig. $1 ; P<0.001$ ).

Reducing the concentration of IA in the controls without Microcystis reduced survival. At the end of the experiment, $100 \%$ IA had the highest survival $(85 \% \pm 3.4)$, while survival in the $50 \%$ IA treatment was lower $(75 \% \pm 2.3$ for E. affinis, $60 \% \pm 8.2$ for P. forbesi) (Fig. 1). Starved copepods (i.e. those fed $0 \%$ IA and $0 \%$ Microcystis) survived for several days, reaching 100\% mortality after 7-9 days for both species, though $P$. forbesi had slightly longer average persistence than E. affinis.

Compared to E. affinis, $P$. forbesi was more sensitive to differences between the Microcystis strains (MC+ versus $\mathrm{MC}-$ ) and had a higher survival rate on a MC+ diet (Fig. 1). Differences in survival of $P$. forbesi between the two different Microcystis strains took several days to emerge. Both strains ( $\mathrm{MC}+$ or $\mathrm{MC}-$ ) caused comparable mortality until day 6 , and thereafter $P$. forbesi mortality on the $\mathrm{MC}-$ diet was greater than that on the MC+ diet (Fig. 1). Towards the end of the experiment, $P$. forbesi mortality began to decline in most of the MC+ diets, but not in the MC- diets (Fig. 1). For treatments with $<50 \%$ Microcystis, the MC- diet killed more $P$. forbesi than the MC+ diet, while these diets caused similar mortality for E. affinis (Fig. 1).

\section{Survival analysis}

The increased proportion of Microcystis in the diet caused higher mortality and increased the probability of mortality (Fig. 2). The effect test showed that diet type had a significant effect on survival and that differences in survival were not because of copepod species (Table 1a). When comparing results among the treatments (Table 1b), differences in survival were caused by the Microcystis strains (MC) and the ratio of Microcystis in food (\% Microcystis). There was a significant difference in how each species responded to the $\mathrm{MC}+$ versus the MC- strains, as well as the ratio of Microcystis in food, as shown by the interaction terms (species $\times \mathrm{MC}$ ) and (species $\times \%$ Microcystis), respectively, in Table $1 \mathrm{~b}$.

Survival/mortality probability tests confirmed that copepods responded differently, both to the individual strains (MC+ versus MC-) and to the ratio of Microcystis in their food. Pseudodiaptomus forbesi was more likely to survive on the MC+ diet compared to an MC- diet when the diet contained $<50 \%$ Microcystis (Fig. 2). In contrast, differences in the Microcystis strain had no significant effect on E. affinis survival at the end of the experiment. Additionally, P. forbesi was more sensitive to changes in the Microcystis content of its diet, as increased Microcystis resulted in higher mortality. In contrast, increasing Microcystis did not significantly change the probability of mortality for E. affinis (Fig. 2). Rather, the presence of Microcystis was more important in reducing E. affinis survival probability by the end of the experiment.

\section{Mean survival time}

The time to $50 \%$ mortality (TL 50) was inversely proportional to the \% Microcystis in diets, but varied significantly with copepod species and Microcystis 
Fig. 1 Survival of Eurytemora affinis and Pseudodiaptomus forbesi on diets containing a mixture of instant algae (IA) and Microcystis (MC+ or MC-), at various proportions of the total food, compared to survival on corresponding control diets containing only IA at the same IA concentration as in the corresponding mixture of IA and Microcystis. $C_{\mathrm{T}}$ shows survival on the total amount of control diet $(100 \%$ IA). Each line represents the mean of a minimum of three replicates $( \pm S E)$.

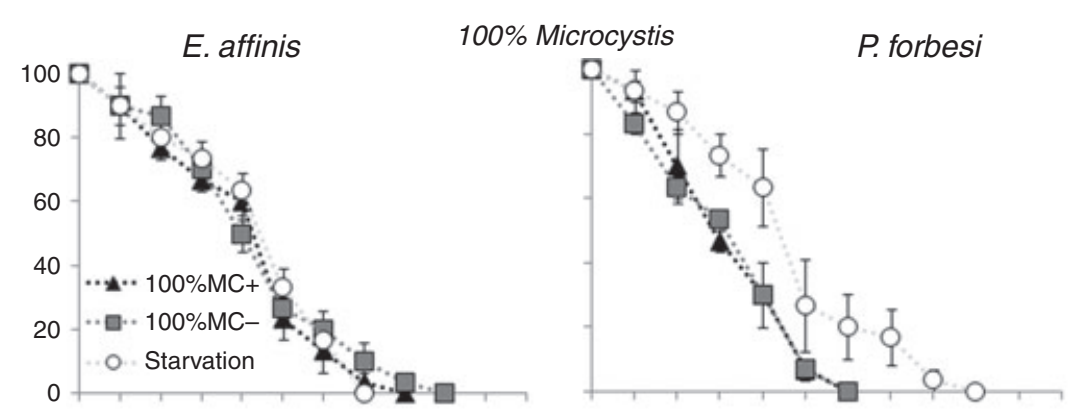

$50 \%$ Microcystis

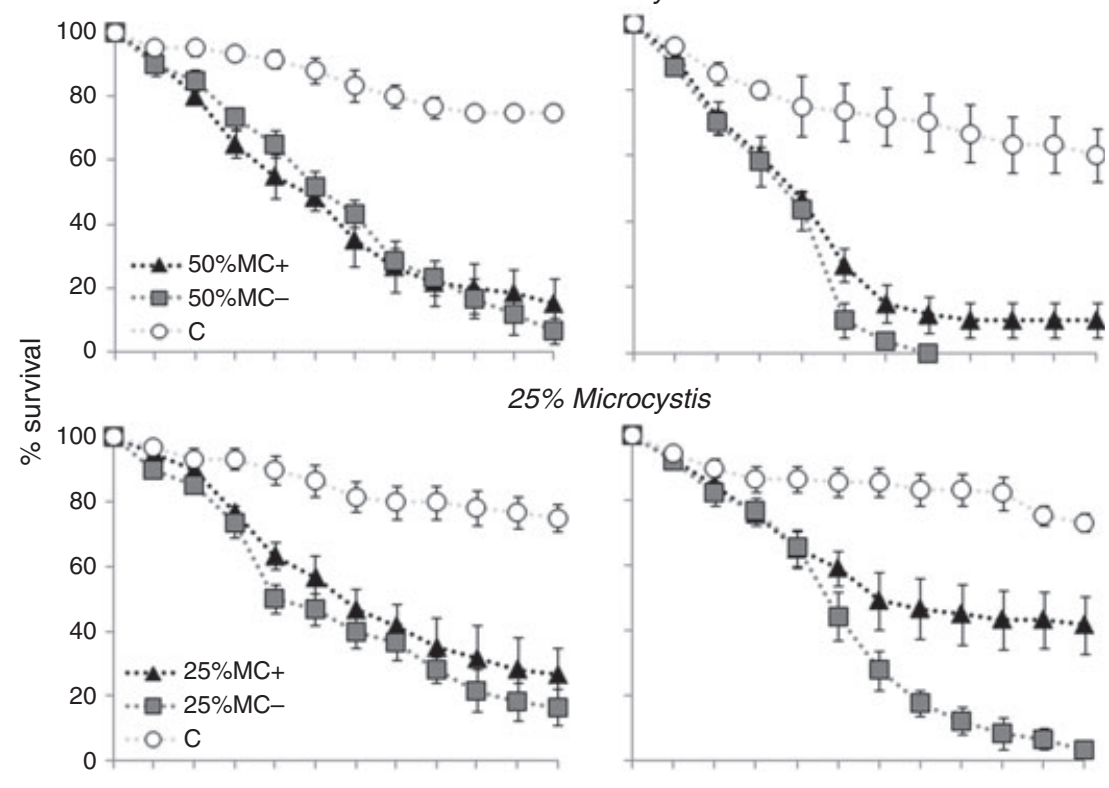

$10 \%$ Microcystis

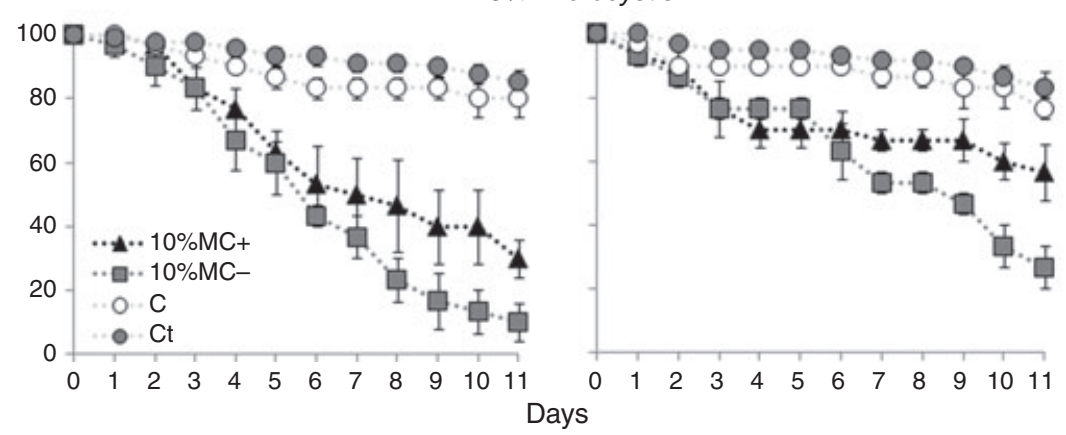

strain (Fig. 3). Among the two copepod species, TL-50 was similar when the ratio of dietary Microcystis was high (50 and 100\%), but diverged at lower ratios of dietary Microcystis (10 and 25\%) (Fig. 3). Two patterns emerged at these lower Microcystis treatments. First, on average, $P$. forbesi lived longer than E. affinis when Microcystis was in the diet. Second, the variation in Microcystis strains caused a significant difference in P. forbesi survival time compared to E. affinis (Fig. 3).
The IA control diets (50, 75, 90 and 100\%) all had TL-50 $>15$ days. Starved copepods had a mean survival time of $3.99 \pm 0.04$ (E. affinis) and $4.23 \pm 0.6$ (P. forbesi) days.

Verification of Microcystis strains, dissolved MC and ingestion

Cross-contamination between the $\mathrm{MC}+$ and $\mathrm{MC}-$ strains of Microcystis was not observed in the 


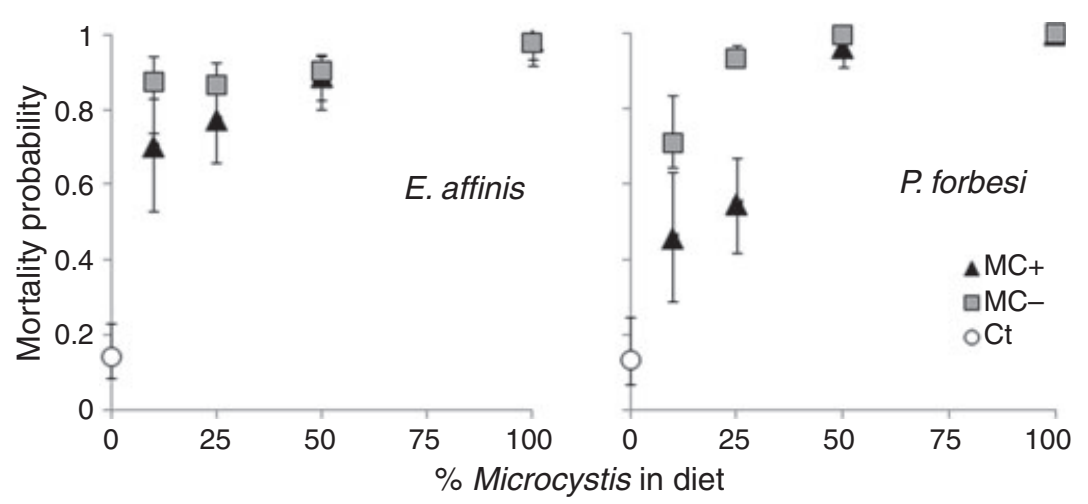

Fig. 2 Probability of copepod mortality in relation to the proportion of Microcystis $(\mathrm{MC}+$ or $\mathrm{MC}-)$ in the diet. Values are based on survival estimates of the parametric model (test of deviance), for the final day of feeding experiment (day 11). For comparison, $C_{\mathrm{T}}$ refers to mortality on the total amount of control diet with no Microcystis (100\% instant algae). Bars indicate $95 \% \mathrm{CI}$.
Table 1 Summary results for the parametric survival fit (test of deviance) and differences in the effect of (a) species and diet (between all diets, controls and Microcystis) on copepod survival probability; results show the factorial interaction of species (Pseudodiaptomus forbesi and Eurytemora affinis) with each diet treatment and (b) the effect of species, MC presence and \% Microcystis on copepod survival probability (excludes control diets); results show the factorial interaction of species ( $P$. forbesi and E. affinis) with $\mathrm{MC}$ presence (MC+ or MC- strain) and \% of Microcystis in the diet (10, 25, 50 and 100\%)

\begin{tabular}{|c|c|c|c|c|}
\hline Source & $\begin{array}{l}\text { No. of } \\
\text { parameters }\end{array}$ & d.f. & $\chi^{2}$ & Prob $>\chi^{2}$ \\
\hline \multicolumn{5}{|l|}{ (a) } \\
\hline Species & 1 & 1 & 0.928 & 0.335 \\
\hline Diet & 12 & 12 & 730.193 & $<0.001$ \\
\hline Species $\times$ diet & 12 & 12 & 42.383 & $<0.001$ \\
\hline \multicolumn{5}{|l|}{ (b) } \\
\hline Species & 1 & 1 & 0.833 & 0.361 \\
\hline MC & 1 & 1 & 22.372 & $<0.001$ \\
\hline$\%$ Microcystis & 3 & 3 & 141.288 & $<0.001$ \\
\hline Species $\times$ MC & 1 & 1 & 8.917 & 0.003 \\
\hline Species $\times \%$ Microcystis & 3 & 3 & 30.938 & $<0.001$ \\
\hline MC $\times \%$ Microcystis & 3 & 3 & 13.236 & 0.004 \\
\hline
\end{tabular}

MC, microcystin.

treatment diets during the trials as verified by periodic measurements of MC-LR concentration with an ELISA. Furthermore, PCR confirmed the presence of Microcystis-specific 16S rDNA genes in both Microcystis strain but, as expected, the MC synthetase genes $m c y \mathrm{~B}$ and $m c y \mathrm{D}$ were detected only in the $\mathrm{MC}+$ strain. Mean cell-bound MC-LR concentration in the $100 \%$ $\mathrm{MC}+$ treatment was $1.62 \mu \mathrm{g} \mathrm{L}^{-1}( \pm 0.18, n=7)$ and $348 \mu \mathrm{g} \mathrm{L}^{-1}( \pm 49, n=8)$ in the MC+ Microcystis culture. This corresponds to an estimated $4.87 \mu \mathrm{g} \mathrm{mgC}^{-1}$ $( \pm 0.98, n=7)$ of MC-LR per Microcystis biomass. The mean cell density in the $100 \%$ Microcystis treatment was 93000 cells $\mathrm{mL}^{-1}( \pm 1458, n=14)$. Dissolved MC-LR was not detected in the treatment beakers during the experiment. Copepods ingested Microcystis in all treatments as observed qualitatively from the fluorescence of phycocyanin.

\section{Discussion}

Our results provide strong evidence that the mortality of both $P$. forbesi and E. affinis was because of dietary Microcystis. As predicted, an increase in the ratio of Microcystis in the diet reduced copepod survival within days, and even the smallest addition to the diet $(10 \%)$ significantly reduced copepod survival. High survival on the control diet suggests that mortality was driven by toxicity from Microcystis and that background mortality was low. Similar toxic effects of dietary Microcystis on zooplankton have often been observed (DeMott et al., 1991; Kirk \& Gilbert, 1992; Wilson et al., 2006). What is unique to this study is the contrast between the two copepod species in their response to the dietary ratio and strains of Microcystis and, more surprisingly, the greater tolerance of $P$. forbesi for the $\mathrm{MC}+$ strain relative to the $\mathrm{MC}-$ strain.

The hypothesis that $\mathrm{MC}+$ cells would cause higher mortality was not supported, as the MC- strain caused similar (E. affinis) or higher (P. forbesi) mortality. Microcystis produces several non-MC metabolites that are toxic when ingested and probably resulted in the harmful effects found here. Examples include lipopolysaccharides, which reduce the efficiency of detoxification, microviridin, which reduces protease activity, and various other unidentified toxins (Kurmayer \& Juttner, 1999; Rohrlack et al., 2004; Wiegand \& Pflugmacher, 2005). As shown with other zooplankton, our results highlight the potential toxicity of non-MC metabolites to copepods (Wilson et al., 2006). 
Fig. 3 Mean time to mortality (TL-50) of each treatment and copepod species as a function of the ratio of Microcystis (MC+ or $\mathrm{MC}-)$ in diet $( \pm \mathrm{SE})$.

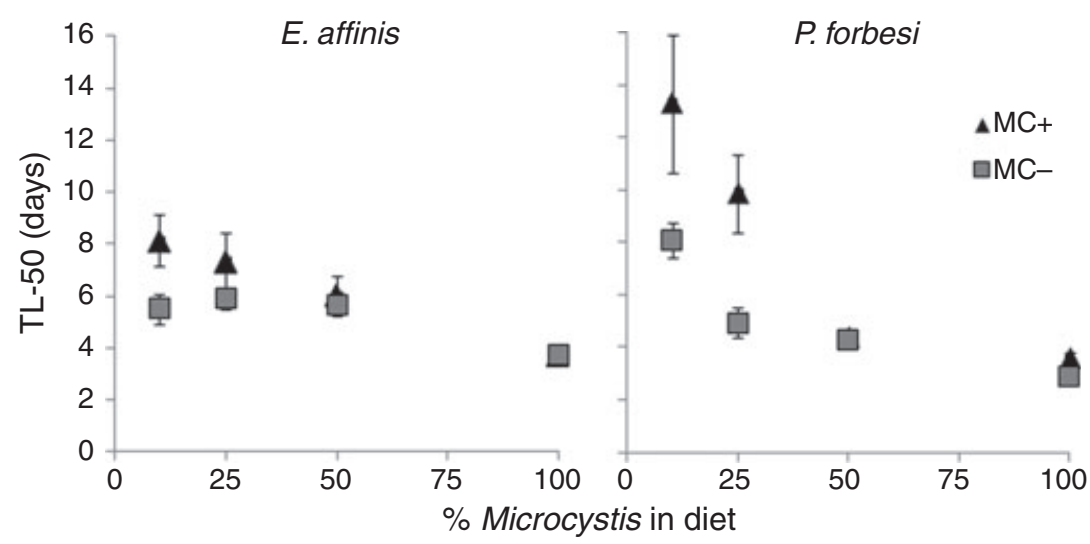

Declining mortality of $P$. forbesi following sustained exposure to the MC+ Microcystis suggests that prolonged exposure may prompt an adaptation to this strain, either via physiological tolerance or a change in feeding behaviour. Species-specific tolerance has ecological significance, since it may explain how some zooplankton can dominate during blooms of toxic algae (Kirk \& Gilbert, 1992; Work \& Havens, 2003; Karjalainen et al., 2007).

Previous exposure to Microcystis over evolutionary timescales may improve physiological tolerance (i.e. detoxification) in zooplankton (DeMott et al., 1991), which may be naturally higher for some copepod species (Kumar, 2003). While increased tolerance of Microcystis in daphniids can also be triggered by short-term exposure, this ability varies among species and the relationship between evolutionary exposure (genotypic effects) and improved detoxification remains unknown (Gustafsson \& Hansson, 2004; Sarnelle \& Wilson, 2005). However, detoxification is a process that follows ingestion and probably not the mechanism by which $P$. forbesi was better able to tolerate MC+ Microcystis. For MC detoxification to occur, $P$. forbesi in the $\mathrm{MC}+$ treatments would first have to ingest the Microcystis cells, thereby ingesting both MC and the non-MC metabolites. In such a case, we would expect to see higher survival for the copepods in the MC- treatments (relative to $\mathrm{MC}+$ ), since MC detoxification causes added stress to zooplankton (Pflugmacher et al., 1998).

Nevertheless, higher mortality in the MC- treatments confirms that the ingestion of non-MC metabolites is indeed lethal, excluding physiological tolerance of $\mathrm{MC}$ as the main cause for coexistence with MC+ Microcystis. A more likely mechanism is selective feeding, which is a common adaptation in copepods exposed to toxic cyanobacteria for extended periods (DeMott \& Moxter, 1991; Kleppel, 1993; Koski, Engstrom \& Viitasalo, 1999). Incidental mortality later in the experiment may be explained by accidental ingestion, as copepods can passively ingest Microcystis cells despite selective feeding (Panosso et al., 2003). High mortality at the onset of exposure to the MC+ diet suggests an acclimation phase when $P$. forbesi optimises MC avoidance and subsequently improves its ability to survive in the presence of Microcystis by feeding selectively on the more palatable food particles.

Assuming that $P$. forbesi can reject harmful particles such as MC+ cells, why does it not also reject the fatal MC- cells with comparable success? Zooplankton have developed different means of detecting and avoiding cyanobacterial toxins, which are considered as evolved defences against zooplankton grazing (DeMott \& Moxter, 1991; DeMott et al., 1991). Although MCs have been shown to deter feeding in daphniids, some copepods rely on more general and unidentified lipophylic toxins to detect and avoid ingesting harmful cyanobacteria (Kurmayer \& Juttner, 1999; Rohrlack et al., 1999). Comparing different calanoid copepods exposed to the cyanobacteria Nodularia, the hepatotoxin nodularin was the grazing deterrent for E. affinis, which selectively fed on nodularin-free cyanobacteria (Engstrom et al., 2000). In contrast, Acartia grazing was deterred by a more general cyanobacterial cue other than nodularin, as it selected against Nodularia regardless of its nodularin content. This is consistent with our results and shows that deterrence of copepod grazing on cyanobacteria is commonly mediated by species-specific chemosensory 
cues. There is no real evidence in the current study showing that differences in the survival of E. affinis and P. forbesi exposed to each Microcystis strain is caused by differences in feeding selectivity. Nevertheless, based on the role of species-specific cues for copepod feeding selectivity, we speculate that MC may be the dominant feeding deterrent for $P$. forbesi, resulting in an ability only to avoid $\mathrm{MC}+$ cells.

Higher survival of $P$. forbesi in the MC+ treatments is surprising and contrary to the presumption of most zooplankton-Microcystis interactions. Typically, the MC content of food either reduces or has no significant effect on survival (Rohrlack et al., 1999; Lurling, 2003; Wilson et al., 2006). Following this general prediction, and in contrast to $P$. forbesi, MC content of Microcystis had no significant effect on E. affinis, as both $\mathrm{MC}+$ and $\mathrm{MC}-$ strains caused similar mortality. Despite such species-specific differences, our results indicate that the non-MC compounds in Microcystis cells can be the main factor causing negative effects on some copepods.

Eurytemora affinis and P.forbesi also responded differently to the proportion of Microcystis in their food, regardless of strain. In general, P. forbesi did better for a given Microcystis diet, and its survival improved with an increased proportion of good food. Calanoid copepods can show reduced ingestion for toxic cyanobacteria when alternative food is available, which probably explains the negative relationship between the ratio of dietary Microcystis and $P$. forbesi survival (DeMott \& Moxter, 1991; Burns \& Hegarty, 1994; Koski et al., 1999). In contrast, E. affinis survival varied much less with the ratio of dietary Microcystis. This may indicate inefficient feeding selection by this species, which was also shown in the Baltic Sea (Engstrom et al., 2000). Pseudodiaptomus forbesi may be better adapted to survive in the presence of Microcystis via feeding selection, and further investigation would help verify the mechanism.

In addition to toxicity, Microcystis is also nutritionally inadequate for zooplankton (DeMott et al., 1991; Christoffersen, 1996). Relatively high survival in the controls indicates that sufficient nutrition was provided by the IA part of the Microcystis - IA mixed diet. Hence, even if Microcystis nutritional deficiency was a significant issue, IA should have sustained survival at least as well as the controls. Since survival with Microcystis is significantly lower than the controls without Microcystis, the toxic effects of Microcystis dominate any potential consequence of nutritional inadequacy. Toxic cyanobacteria can also harm zooplankton by inducing feeding inhibition (DeMott et al., 1991; Rohrlack et al., 1999; Lurling, 2003; Wiegand \& Pflugmacher, 2005). However, this is unlikely for calanoid copepods that avoid lower quality food particles without reducing their ingestion rate on high-quality food (DeMott \& Moxter, 1991; Kleppel, 1993; Koski et al., 1999; Tackx et al., 2003). Surviving copepods in Microcystis treatments were actively swimming, had full guts and continued to produce faecal pellets, indicating that copepods continued ingestion during the experiment. Both strains of Microcystis and the IA controls were uni- and bi-cellular, ruling out any potential effects from colonial morphology, which can also hinder ingestion (Fulton \& Paerl, 1987; Ghadouani, Pinel-Alloul \& Prepas, 2003).

Empirical evidence from the field for the toxic effects of cyanobacteria on zooplankton is less common and typically muted in comparison to laboratory interactions, where variables can be strictly controlled (Ferrao-Filho \& Azevedo, 2003; Work \& Havens, 2003). Ecosystem impacts of cyanobacteria at larger scales are more subtle because of stochastic variability, species-specific adaptations, colonial morphology, multiple trophic links and unpredictable food web dynamics (Kozlowski-Suzuki et al., 2003; Rohrlack et al., 2004; Wilson et al., 2006; Beninca et al., 2008). However, the impacts of cyanobacteria at the ecosystem scale have been demonstrated, and include the disruption of the benthic food web via bioaccumulation of toxins, shifts in zooplankton and phytoplankton species composition, and possible indirect effects shifting the pelagic community towards the microbial loop (Fulton \& Paerl, 1987; Christoffersen, 1996; Karjalainen et al., 2007).

We found strong evidence that dietary Microcystis reduces copepod survival even at low dietary ratios, suggesting possible direct impacts on copepods in the San Francisco Estuary where Microcystis seasonally dominates phytoplankton (Lehman et al., 2005). During blooms in the estuary, Microcystis dominates the phytoplankton community and its density fluctuates, between about $0 \times 10^{3}$ and $2 \times 10^{3}$ cell $\mathrm{mL}^{-1}$ in the euphotic zone, depending on the location and time, though densities up to $2.2 \times 10^{6} \mathrm{~mL}^{-1}$ have been recorded (Lehman et al., 2008). The average range monitored in the estuary corresponds with the 
treatment diets in this study, at least in terms of cell density, as the highest concentration of Microcystis fed to copepods was $9.3 \times 10^{3} \mathrm{~mL}^{-1}$. During blooms, persistent concentrations of Microcystis between $10^{3}$ and $10^{6}$ cell $\mathrm{mL}^{-1}$ are often observed in eutrophic waters globally, making the results of this study relevant to the zooplankton community of the San Francisco Estuary as well as other regions (Chorus \& Bartram, 1999; Rinta-Kanto et al., 2005; Costa et al., 2006).

Nevertheless, as mentioned previously, Microcystis impacts measured in the laboratory may not always represent natural processes in the field. Depending on the intensity of the bloom and the availability of alternative food, including microbial sources such as ciliates, selectively feeding copepods may or may not persist in the presence of Microcystis (DeMott \& Moxter, 1991; Work \& Havens, 2003). Zooplankton in the San Francisco Estuary can be limited by low phytoplankton abundances (Jassby, Cloern \& Cole, 2002; Muller-Solger et al., 2002), and such food limitation can intensify the negative impacts of cyanobacteria on copepods by reducing the effectiveness of selective feeding (DeMott \& Moxter, 1991; Engstrom et al., 2000). Conversely, natural blooms of Microcystis, including in the estuary, are typically dominated by colonial forms (Lehman et al., 2005; Wilson et al., 2006), and copepods are probably more effective at selective feeding in the presence of colonies, when the particle size is larger (Tackx et al., 2003). However, copepods in the San Francisco Estuary accumulate MC during Microcystis blooms, suggesting that ingestion in the field occurs, despite the dominance of colonies (Lehman et al., 2008). For these reasons, although we found negative impacts of Microcystis on copepods, prediction of the ecological impacts must also be informed by coupled laboratory and in-situ field observations of ingestion and food limitation and evaluation of the ability of copepod species to detect and avoid harmful cells.

\section{Acknowledgments}

We greatly appreciate Ida Flores for assistance with culturing copepods and survival experiments, Dr Tomofuri Kurobe for help with development of the PCR, Dr Monika Winder for assistance with epifluorescence microscopy, Dr Emilio Laca for help with statistical analysis and the suggestions of the two anonymous reviewers, which improved the manuscript considerably. Funding of this study is supported by Dr Swee Teh's Aquatic Toxicology Program fund and partially by the California Interagency Ecological Program Pelagic Organisms Decline (IEPPOD), CALFED (project\# PO685515) and California Department of Water Resources (Contract Nos. 4600007499 and 4600008137).

\section{References}

Beninca E., Huisman J., Heerkloss R., Johnk K.D., Branco P., Van Nes E.H., Scheffer M. \& Ellner S.P. (2008) Chaos in a long-term experiment with a plankton community. Nature, 452, 822-826.

Burns C.W. \& Hegarty B. (1994) Diet selection by copepods in the presence of cyanobacteria. Journal of Plankton Research, 16, 1671-1690.

Chorus I. \& Bartram J. (1999) Toxic Cyanobacteria in Water: A Guide to Their Public Health Consequences, Monitoring, and Management. E \& FN Spon, London, U.K.

Christoffersen K. (1996) Ecological implications of cyanobacterial toxins in aquatic food webs. Phycologia, 35, 42-50.

Costa I.A.S., Azavedo S.M.F.O., Senna P.A.C., Bernardo R.R., Costa S.M. \& Chellappa N.T. (2006) Occurrence of toxin producing cyanobacteria in a Brazilian semiarid reservoir. Brazilian Journal of Biology, 66, 211-219.

DeMott W.R. \& Moxter F. (1991) Foraging on cyanobacteria by copepods: responses to chemical defenses and resource abundance? Ecology, 75, 1820-1834.

DeMott W.R., Zhang Q.X. \& Carmichael W. (1991) Effects of toxic cyanobacteria and purified toxins on the survival and feeding of a copepod and three species of Daphnia. Limnology and Oceanography, 36, 13461357.

Engstrom J., Koski M., Viitasolo M., Reinikainen M., Repka S. \& Sivonen K. (2000) Feeding interactions of the copepods Eurytemora affinis and Acartia bifilosa with the cyanobacteria Nodularia. Journal of Plankton Research, 22, 1403-1409.

Ferrao-Filho A.S. \& Azevedo S.M. (2003) Effects of unicellular and colonial forms of toxic Microcystis aeruginosa from laboratory cultures and natural populations on tropical cladocerans. Aquatic Ecology, 37, 2335.

Fulton R.S. \& Paerl H.W. (1987) Toxic and inhibitory effects of the blue-green alga Microcystis aeruginosa on herbivorous zooplankton. Journal of Plankton Research, 9, 837-855.

Ghadouani A., Pinel-Alloul B. \& Prepas E.E. (2003) Effects of experimentally induced cyanobacterial 
blooms on crustacean zooplankton communities. Freshwater Biology, 48, 363-381.

Gustafsson S. \& Hansson L.-A. (2004) Development of tolerance against toxic cyanobacteria in Daphnia. Aquatic Ecology, 38, 37-44.

Horning W.B. \& Weber C.I. (1985) Short-term Methods for Estimating the Chronic Toxicity of Effluents and Receiving Waters to Freshwater Organisms. pp. 58-75. EPA/600/4/85/014, USEPA, Cincinnati, OH, U.S.A.

Jassby A.D., Cloern J.E. \& Cole B.E. (2002) Annual primary production: patterns and mechanisms of change in a nutrient rich tidal ecosystem. Limnology and Oceanography, 47, 698-712.

Karjalainen M., Engstrom-Orst J., Korpinen S., Peltonen H., Paakonen J.-P., Ronkkonen S., Suikkanen S. \& Viitasalo M. (2007) Ecosystem consequences of cyanobacteria in the Northern Baltic Sea. Ambio, 36, 195-202.

Kirk K.L. \& Gilbert J.J. (1992) Variation in herbivore response to chemical defenses: zooplankton foraging on toxic cyanobacteria. Ecology, 73, 2208-2217.

Kleppel G.S. (1993) On the diets of calanoid copepods. Marine Ecology Progress Series, 99, 183-195.

Koski M., Engstrom J. \& Viitasalo M. (1999) Reproduction and survival of the calanoid copepod Eurytemora affinis fed with toxic and non-toxic cyanobacteria. Marine Ecology Progress Series, 186, 187-197.

Kozlowski-Suzuki B., Karjalainen M., Lehtiniemi M., Engström-Öst J., Koski M. \& Carlsson P. (2003) Feeding, reproduction and toxin accumulation by the copepods Acartia bifilosa and Eurytemora affinis in the presence of the toxic cyanobacterium Nodularia spumigena. Marine Ecology Progress Series, 249, 237-249.

Kumar R. (2003) Effect of different food types on developmental rates and demographic parameters of Phyllodiaptomus blanci (Copepoda:Calanoida). Archiv für Hydrobiologie, 157, 351-377.

Kurmayer R. \& Juttner F. (1999) Strategies for the co-existence of zooplankton with the toxic cyanobacterium Planktothrix rubescens in Lake Zurich. Journal of Plankton Research, 21, 659-683.

Lehman P.W., Boyer G., Hall C., Waller S. \& Gehrts K. (2005) Distribution and toxicity of a new colonial Microcystis aeruginosa in the San Francisco Bay Estuary, California. Hydrobiologia, 541, 87-99.

Lehman P.W., Boyer G., Satchwell M. \& Waller S. (2008) The influence of environmental conditions on the seasonal variation of Microcystis cell density and microcystins concentration in San Francisco Estuary. Hydrobiologia, 600, 187-204.

Lurling M. (2003) Daphnia growth on microcystin-producing and microcystin-free Microcystis aeruginosa in different mixtures with the green alga Scenedesmus obliqus. Limnology and Oceanography, 48, 2214-2220.
Muller-Solger A., Jassby A.D. \& Muller-Navarra D. (2002) Nutritional quality of food resources for zooplankton (Daphnia) in a tidal freshwater system (Sacramento-San Joaquin River Delta). Limnology and Oceanography, 47, 1468-1476.

Ouellette J.A. \& Wilhelm S. (2003) Toxic cyanobacteria: the evolving molecular toolbox. Frontiers in Ecology and the Environment, 1, 359-366.

Paerl H.W. (1988) Nuisance phytoplankton blooms in coastal, estuarine, and inland waters. Limnology and Oceanography, 33, 823-847.

Panosso R., Carlsson P., Kozlowsky-Suzuki B., Azevedo S.M.F.O. \& Graneli E. (2003) Effect of grazing by a neotropical copepod Notodiaptomus, on a natural cyanobacterial assemblage of toxic and non-toxic cyanobacterial strains. Journal of Plankton Research, 25, 1169-1175.

Pflugmacher S., Wiegand C., Oberemm A., Beattie K.A., Krause E., Codd G. \& Steinberg C.E.W. (1998) Identification of an enzymatically formed glutathione conjugate of the cyanobacterial hepatotoxin microcystin-LR: The first step of detoxification. Biochimica et Biophysica Acta, 1425, 527-533.

Reynolds C.S. \& Jaworski G.H.M. (1978) Enumeration of natural Microcystis populations. British Phycological Journal, 13, 269-277.

Rinta-Kanto J.M., Ouellette A.J.A., Boyer G.L., Twiss M.R., Bridgeman T.B. \& Wilhelm S.W. (2005) Quantification of toxic Microcystis spp. during the 2003 and 2004 blooms in Western Lake Erie using quantitative real-time PCR. Environmental Science and Technology, 39, 4198-4205.

Rohrlack T., Dittman E., Borner T. \& Christoffersen K. (1999) Effects of cell-bound microcystins on survival and feeding of Daphnia spp. Applied and Environmental Microbiology, 67, 3523-3529.

Rohrlack T., Christoffersen K., Kaerbernick M. \& Neilan B.A. (2004) Cyanobacterial protease inhibitor Microviridin J causes a lethal molting disruption in Daphnia pulicaria. Applied and Environmental Microbiology, 70, 5047-5050.

Sambrook J. \& Russell D.W. (2001) Molecular Cloning: A Laboratory Manual, 3rd edn. Cold Spring Harbor Laboratory Press, Cold Spring Harbor, NY, USA.

Sarnelle O. \& Wilson A.E. (2005) Local adaptation of Daphnia pulicaria to toxic cyanobacteria. Limnology and Oceanography, 50, 284-289.

Sommer T., Armor C., Baxter R. et al. (2007) The collapse of pelagic fishes in the upper San Francisco Estuary. Fisheries, 32, 270-277.

Tackx M.L.M., Herman P.J.M., Gasparini S., Irigoien X., Billiones R. \& Daro M.H. (2003) Selective feeding of Eurytemora affinis (Copepoda, Calanoida) in temperate 
estuaries: model and field observations. Estuarine and Coastal Shelf Science, 56, 305-311.

Turriff N., Runge J.A. \& Cembella A.D. (1995) Toxin accumulation and feeding behaviour of the planktonic copepod Calanus finmarchicus exposed to the red-tide dinoflagellate Alexandrium excavatum. Marine Biology, 123, 55-64.

Wiegand C. \& Pflugmacher S. (2005) Ecotoxicological effects of selected cyanobacterial metabolites: a short review. Toxicology and Applied Pharmacology, 203, 201218.
Wilson A.E., Sarnelle O. \& Tillmanns A.R. (2006) Effects of cyanobacterial toxicity and morphology on the population growth of freshwater zooplankton: metaanalyses of laboratory experiments. Limnology and Oceanography, 51, 1915-1924.

Work K.A. \& Havens K.A. (2003) Zooplankton grazing on bacteria and cyanobacteria in a eutrophic lake. Journal of Plankton Research, 25, 1301-1307.

(Manuscript accepted 11 November 2009) 